\title{
Brief Analysis on Design Ingenuity and Culture of "Duobaoge" of Qing Dynasty Palace
}

\author{
Chao Zheng \\ School of Art Design \\ Shanghai University of Engineering and Science \\ Shanghai, China 201620
}

\author{
Wei Zhu \\ School of Art Design \\ Shanghai University of Engineering and Science \\ Shanghai, China 201620
}

\begin{abstract}
As a court appreciation and collection tool, Duobaoge reflects craftsmanship and aesthetic taste of palace of the Qing Dynasty. It is referred to as model of archaic packaging design. It has complex craftsmanship and ingenious structure. Articles for amusement it stores can be mutually combined. It is not only blended in harmony in terms of functionality and appreciation, but also beneficial to collection and storage and easy to carry. The traditional idea of hiding ingenuity in simpleness runs through it, reflecting a kind of mature appreciation and collection concept of Qing Dynasty.
\end{abstract}

\section{Keywords—Qing Dynasty Palace; Duobaoge; design; culture}

\section{INTRODUCTION}

Appearance of Duobaoge in Qing Dynasty palace is to make it convenient for emperor to appreciate and play priceless treasures in the palace at any time. With systematic political management, there are various types of storeroom in the palace, such as department of supplies under imperial household department in Qing Dynasty palace consists of silver, satin, clothing, fur, china and tea library, six in total. Contributive priceless treasures from the world are all collected in these libraries with concierge management. Even if the emperor wants to appreciate rare curios, there still are many procedures. Thus, it is very inconvenient. It is exactly under this situation that people start to make Duobaoge. Duobaoge means storing several or even hundreds of rare curios together in a packaging case which is not over-sized.

\section{OVERVIEW OF DUOBAOGE}

In many documents and archives, Duobaoge is referred to as a hundred articles, a hundred or so pieces, a hundred events and a hundred types and so on. Although there are differences in used words, there is no contradiction in meaning. They all mean many, varied and trivial. Seeing from real objects, these wares are generally in a square case which is not large. The packaging is exquisitely designed. There are boxes inside the case, drawers inside the box, and grids in the drawer. Each grid contains a piece of rare curio (or exquisite practical small goods). This kind of case can contain several or more than ten pieces at least and hundreds of pieces at most. Rare curios inside the case can be of the same type, for instance, they can all be jade article. But in most cases, it is full of beautiful and numerous things and combines various materials. Precious collectables in Qing Dynasty palace are mainly scroll of calligraphy and painting, album, jade ware, chinaware, bronze ware, enamel, lacquer ware, bamboo engraving, wood craving, ivory, moulded gourd-shaped articles and so on.

\section{A. Differentiation of Precious Articles}

Then, what is the relation between Duobaoge and "a hundred articles"? Actually we all know that connotation and extension of words are dynamic, so it is necessary to conduct specific analysis in specific context. In general, "a hundred articles" has a wider meaning. A hundred articles originally is the joint name of numerous and jumbled rare curios and referred to as a proper term for integrator of them directly later on. It should be mentioned that significance of "a hundred articles" does not lie in creation of new cultural relic varieties, but lie in reflection of a kind of unique appreciation and collection concept and creative space structure mode. A hundred articles can cover all the previously mentioned names sometimes, but there are differences and even difference of degree sometimes. For instance:

- On lunar January 4th, the third year of Qianlong administration (1738), Gaozong issued a decree: Store 60 bronze seals of first class in Duobaoge and 20 bronze seals of second class in "a hundred events".

- On lunar December 1st, the sixth year of Qianlong administration (1741), a decree was issued to invite people to see a small $\mathrm{Ru}$ glaze flower receptacle and ask them: Can it be stored in Duobaoge? The next day, Fang Xihua judged: It belongs to first class and can be stored in Duobaoge.

- On lunar November 20, the ninth year of Qianlong administration (1744), Yang Qiyun discriminated a Dingyao melon-shaped teapot in a hundred types fine jade case and thought that it was beautiful and could be stored in Duobaoge; However,on lunar January 5, the next year, Samuha, a leader of seven grades, rechecked on it and defined it as common thing, thus it could not enter Duobaoge. Therefore, Gaozong issued a decree: Although it can not enter Duobaoge, it can still be stored in a hundred articles. 1 
Seeing from these historical data, it seems that Duobaoge and a hundred types are different as to superiority or inferiority. Compared with a hundred types, Duobaoge has a higher grade.

\section{B. Source of Craftsmanship}

If we want to trace the source of craftsmanship tradition of Duobaoge, it can be related to double layer nine son casket box unearthed in No.1 Han Dynasty tomb of Mawangdui, Changsha, Hunan "Fig. 1". Some scholars point out that the lacquer box unearthed in Chu State tombs of the Warring States period in Baoshan, Jinmen, Hubei, consists of six grids respectively for ear cup, square pot and square plate. It is matched exactly with reasonable structure and it is in the early form of Duobaoge. However, portable stationery case created by later Ming Dynasty people has direct influence on design of a hundred articles. Gao Lian's To Obey Lives Eight Notepaper (Volume Eight) records a piece of "possession case", which is almost the same as recordation in "tour memo" of Tu Long's Kao Pan Yu Shi (Volume Four). They are both used in "mountain tour". As data displays, stored goods are mainly practical, and are mainly censer, washing, tea bowel, books and so on. In comparison, recordation in Wen Zhenheng's Treatise on Superfluous Things is mainly scholar's treasure playing thing, like archaic jade, ancient bronze paperweight and Song Tihe. He also especially emphasizes on "Exquisite and elegant antique can all be stored for appreciation", which is very close to goods combination thought of Duobaoge in later ages. Therefore, more scholars think that the so called "preparation box" or "smooth way case" in art essay of Ming Dynasty can both be regarded as rudiment and predecessor of Duobaoge in Qing Dynasty palace.

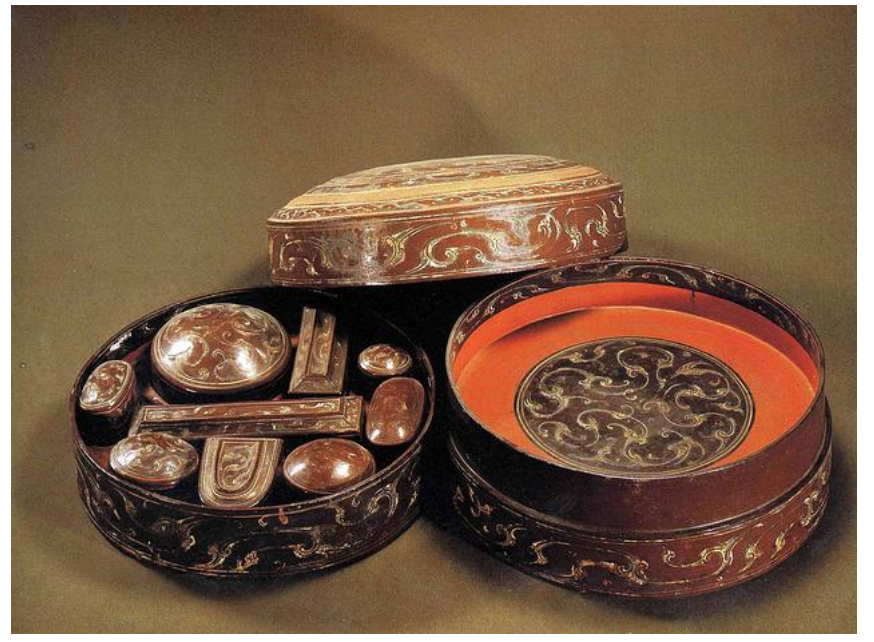

Fig. 1. Double layer nine son casket box unearthed in No.1 Han Dynasty tomb of Mawangdui, Changsha

\section{INGENIOUS DESIGN IDEA OF DUOBAOGE}

Attractiveness in packaging of Duobaoge lies in ingenious structure and refined design. Among packaging of various shaped ware structures in ancient China, Duobaoge may be called supreme model works.

Duobaoge with the simplest structure is hamper shaped, namely a shallow wall box with box cover. For instance, "The
Yearling" Duobaoge of Ming Dynasty only has simple interlayer changes without a high box body. It is clear at a glance, therefore its content configuration is usually pleasing to eyes with generosity sense.

In addition, some Duobaoge boxes have two-layer drawers or three-layer drawers and these drawers share one box cover or a packaging case. Drawer type Duobaoge is also common to see. It is in regular symmetry and unbearable for eyes, but its content is usually the most abundant. For instance, a piece of "craved red lacquer packaging case in Jiajing period of Ming Dynasty" has ten drawers and stores 108 cultural relics. Covers are different due to different design layouts of drawer and ten drawers equal to rich changes of ten Duobaoge. The above cases and boxes mostly belong to plane and open type. Although there is interlayer or concealed grid, it emphasizes on visible objects from a bird's eye view in front. Other attractive modelings mainly are:

\section{A. Side Appreciation Type}

This kind of Duobaoge is mainly relatively deep box with empty craved side wall to form various types of hollow pane, through which people can see implements on lower layer inside the box, thus increasing appreciated space of the first sight. For instance, positive side of a porcelain embedded Duobaoge with ancient figures is dealt in this way; Taking red sandalwood for another instance, apart from rare curios stored at front top and in middle lower layer, hollow orifice in various types on both sides also stores a dozen or so playing objects. Hollowing board on both sides can be pulled open towards side edge.

\section{B. Ingenious Variant Crankshaft Type}

This kind of Duobaoge uses crankshaft connection in structural craftsmanship and connects several small grids into a whole. For instance, "Qing Dynasty bamboo filament branch tied lotus Duobaoge" round packaging box uses firstly bamboo filament jointing and secondly branch tied lotus pattern tabasheer piece decoration for its ektexine and uses crankshaft principle to divide the cylindrical box into four sectors, which can form an in-line screen by $180^{\circ}$ open and become square tubular by $360^{\circ}$ turning over. Each sector consists of many lattice layers, among which cylindrical lattice layer can not only be divided into several grids, but also support $360^{\circ}$ rotation. The whole box shows craftsman's ingenuity and optimized design ability. There are 27 small articles for amusement stored in lattice layer of this round box. Besides ancient and Qing Dynasty jade wares, there are also painter's paintings from Qianlong period's imperial palace of Qing Dynasty, hand scroll and album. Triangle drawer in the lowest layer of each sector should contain one piece of hand scroll. There are only three pieces at present, which respectively are "Fang Cong drawing landscape", "Yang Dazhang drawing flowers and plants" and "Li Bingde drawing flowers and plants". They are just seven centimeters lengthwise. A figure album drawn by Jin Tingbiao is stored in a sector's middle triangle drawer and its width and length are just three centimeters. It can be said that they are the most compact hand scroll and album collected in national palace museum with precious value. This kind of Duobaoge packaging can be listed 
as palace boutique packaging with the most variant appearance "Fig. 2".

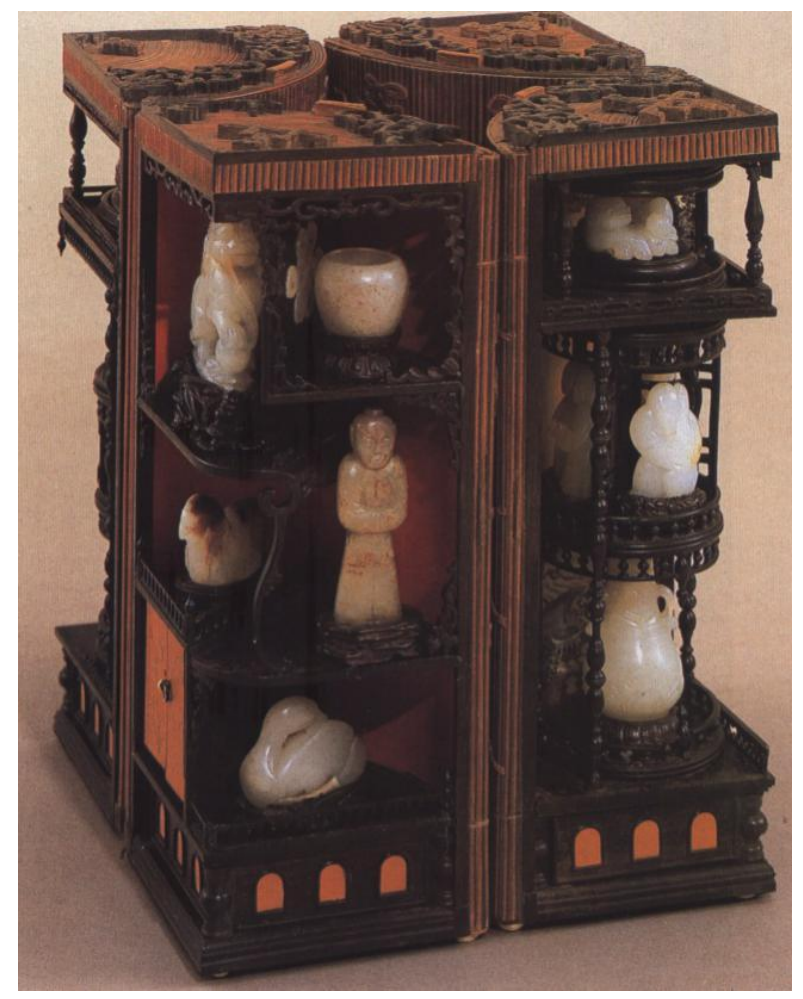

Fig. 2. Qianlong period bamboo filament branch tied lotus Duobaoge round box

\section{All-round Appreciation Type}

Generally, this kind of Duobaoge is not large, but it can be appreciated from all sides. For instance, a genuine small Duobaoge with inscription of "Treasure on the mat", is just six to seven CM long and wide. Thus, it can really be put on mat for appreciation of various rare curios from all sides.

Each cultural relic in Duobaoge has a proper seat and grid. Some even have proper matched cushions at connector port. If the grid is not high enough or superficially seen rare curio is not thick enough, there must be interlayers and blank doors. Some interlayers are separated by chased clapboard while some needs to be drawn out towards side internal layer from a grid near it. Some blank doors are installed outside Duobaoge while some are connected by tenon and can be drawn out towards internal side. In most cases, there is interlayer for storing objects at the bottom of Duobaoge. For instance, a red sandalwood Duobaoge packaged drawer stores a grid of jade cicada, whole pedestal is also a grid which can be pulled open. Inside the grid, there is a white jade small box of Lu Zigang type. Besides, the box generally stores collected objects. Sometimes a small box is covered with a big box and there is brocade cloth cover in the small box. It may be said layers of packaging and extreme precision.

Besides structure, what makes people curios is that Duobaoge adopts what kind of combination procedures to flawlessly deal with and completely match objects and container and whether firstly choose rare curio or structural space. The answer is that these two things combine into one in the process. Under the theme, firstly collect a batch of implements to construct main body of a hundred articles, consider layout and constitution at the same time, and start designing interior space after renovating, seat matching or packaging of each alternative implement. The solution to surplus open space is re-selecting objects according to their size or re-making. Some miniature scroll paintings and albums are exactly tailor-made for this requirement. Seeing from this aspect, the concept of "series packaging" has been imbedded in design sense of artisans in royal workshop at that time. Planning, design and production of Duobaoge have contained integration packaging design idea advocated at present.

\section{Culture of DuObaoge}

\section{A. Palace Culture Under Emperor's Love}

Producing heat of Duobaoge in Qing Dynasty palace certainly is inseparable with emperor's love and personal involvement. According to record in Handicraft Work Record of Imperial Household Department Workshop, on lunar January 27 of the First Year of Yongzheng Dynasty (1723), Shizong issued a decree: "Bring original a hundred articles displayed in hall of mental cultivation here for me to see." 2 Among them, there is a brocade box handed in to royal workshop on lunar January 8 of the 57th year of Kangxi Dynasty (1718), inside which there is a Duobaoge, 188 pieces in total. It is thus clear that design and display of Duobaoge have begun in palace at least in later period of Kangxi Dynasty.

In Qianlong Dynasty, due to enthusiasm of Gaozong, Duobaoge has a more irreplaceable place in palace's manufacture system. Especially in yearly years of his administration, among a myriad of state affairs, whenever Gaozong is free, he will appreciate newly made Duobaoge. In the 9th year of Qianlong Dynasty (1744), he issued a decree: "Submit currently made a hundred articles for reading on the 5th and 10th day, and on the 29th day of a small month." Examination in every five days shows great importance. Moreover, he concerns himself with the whole process of design and producing. For instance, a hundred pieces "fine jade case" starts from recording of its name in "case works" on lunar March 29, the third year of Qianlong Dynasty (1738) and it experiences modifying even on lunar August 10, the 13th year (1748), Gaozong writes subjection: Yu Xing, palace painter from "Ruyi Guan" paints sector type painting for small rosewood grid inside it. It seeks perfection through 10 years of repeated modifying. Sometimes, Gaozong even arranges layout of implements in a hundred articles on his own and he enjoys the process of design. For instance, a hundred pieces "gem case" stores 498 jade articles in total. He puts them in position and specially issues a decree: "Change to wooden tire groove according to my form and there should be no disorder." Excellent craftsmen helping him realize his imagination also win favor. Feng Qi, a tooth artisan from Jiading, becomes one of the most trusted craftsmen with a leap in early years of Qianlong Dynasty, although he does not have a high status in Yongzheng Dynasty. The sign includes assigning him to make a sweet stone dish for "a hundred articles for going out" on lunar June 17, the 6th year of Qianlong Dynasty (1741). The 
records of various relevant information that take "Duobaoge" as collection outline appear in handicraft work archives from the 21st year to the 33rd year of Qianlong Dynasty, which can prove that design and producing of this kind of implements are still very frequent at that time "Fig. 3".

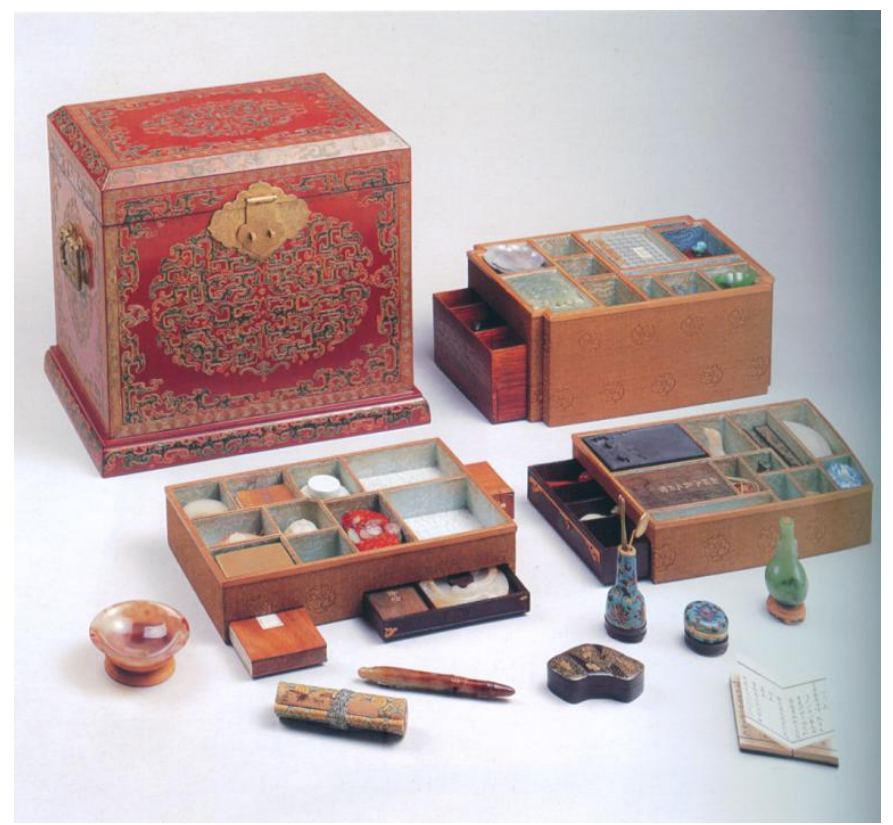

Fig. 3. Qing Dynasty Qianlong period gold lacquer dragon and phoenix pattern Duobaoge

\section{B. Appreciation and Collection Culture under Flourish of Articles for Amusement}

Powerful national strength, people's peaceful and happy livelihood, and full flourish of writing style appear in early period of Qing Dynasty. In Qianlong period, people are earnest about elegance. Inclinations not only include calligraphy, painting and Ding Yi, but also include bamboo and dentale, gold and silver and crystal jade, exquisitely made works and natural wonders. Articles for amusement of more than three generations and of Ming and Qing Dynasties can also be stored in Duobaoge. At ordinary times, Duobaoge in Qing Dynasty palace is usually placed in hall of mental cultivation, palace of gathering excellence, heavy China palace and other daily living places of emperor and queen to make it convenient to open the case to appreciate. Packaging structure of Duobaoge is very ingenious. Opening and closing of packaging is the most changeful. Many structures' producing and intrigue's setting particularly emphasize on interest. This form of packaging considers psychological need of users, making emperors fascinated. Most of currently existed Duobaoge is made under requirement of emperors before Jiaqing. But seeing from some remaining dockets in Duobaoge, there is also small quantity producing in Daoguang, Xianfeng and Tongzhi period.

Wrappage in Duobaoge is "gathering together at all times and in all over the world". Generally in a Duobaoge, there can be jade ware of Shang Dynasty, bronze ware of Han Dynasty, china ware of Song Dynasty, Ruyi of Qing Dynasty, pocket watch from Britain, gold coin from Russia and so on.
Packaging is mainly rare curio. As long as it is proper in volume, it can be shown in Duobaoge in an interesting way. ${ }^{4}$ Design of Duobaoge also considers that most of its packaging is rare curio for amusement, so articles are hidden secretly, there are twists and turns in seeking and appreciator can find another secret drawer interlayer out of their imagination. This kind of exploration fun is clearer in design of Duobaoge.

\section{Ingenious and Exquisite Design Culture}

Interestingness of Duobaoge originates from ingenious design. Qing Dynasty emperors have great interest in Duobaoge. Handicraft Work File of Royal Workshops in Imperial Household Department displays that, Qianlong emperor even takes part in the whole process of design and placement of some rare curio cases. Another file records that, the emperor asks royal workshop to show him the Duobaoge in assembly which is not finished yet every five days in the 9th year of Qianlong Dynasty.

It is not only due to priceless value of rare curios collected in Duobaoge. More importantly, implementation of "cleverness" in design of Duobaoge reaches the peak of perfection. Craftsmen make each space with different width, length and height at random inside each grid through accurate calculation of volume. Open the case and you will see rare curios of different sizes displayed in different spaces. They match exactly without wasting space; once closing up, it is just a well-disciplined ordinary box. Seeing from appearance, it is impossible to see the heaven and earth hidden in it.

In addition, apart from design of window and pedestal in "red sandalwood Duobaoge square case", Qianlong emperor has a "bamboo filament branch tied flowers Duobaoge original case". It uses crankshaft principle in producing and divides the cylindrical box into 4 sectors, which can form a small in-line screen if opening up 180 degrees and form a square tubular if turning over 360 degrees. Each sector consists of many lattice layers. Cylindrical lattice layer not only can be divided into several grids, but also can rotate 360 degrees.

Tier upon tier design inside Duobaoge makes some opening and closing intrigues hidden in lower layer. Just like a game, only when taking objects away, can you "break off the intrigue". It is said that some ingenious designed Duobaoge can make people know and even see inside the grid. There is treasure in drawer or on clapboard. However, you can not get treasure without finding the intrigue.

There is another feature of Duobaoge in Qing Dynasty palace, which is appreciation fun before and after picking and placement. Generally speaking, the bottom of a case will be empty if you take an object out of the case. But Duobaoge has an "intrigue" here. For instance, open Qianlong's red sandalwood dragon and cloud patter Duobaoge square case, and you can find a tray containing jade ware in uppermost layer "Fig. 4". After taking out jade ware, people can surprisingly find that there are not only characters of $\mathrm{Yu}$ Minzhong, minister of imperial college of inscriptions but also paintings from Yang Dazhang, a royal painter. These characters and paintings still have very bright colors which do not fade away with time passing. Without taking out the object, 
you can not find painting and calligraphy hidden at the bottom or you even can not find another piece of cultural relic.

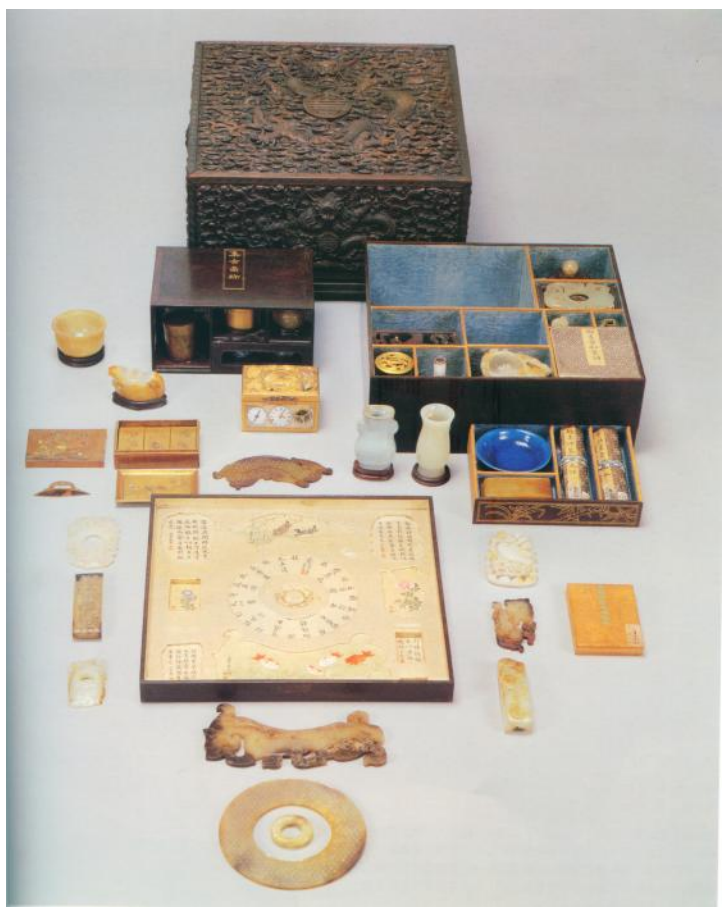

Fig. 4. Qianlong's red sandalwood dragon cloud pattern Duobaoge square box in Qing Dynasty

\section{CONCLUSION}

At last, we will briefly conclude design features and cultural connotation of Duobaoge in Qing Dynasty palace according to real object and record.

- Firstly, it is on the basis of royal household collection, relies on powerful system of palace producing, serves requirement of royal household, reflects taste and aesthetics of emperors and is a miniature representative of palace process design;

- Secondly, it represents a kind of mature appreciation and collection concept. Each rare curio needs to experience selection, classification and gradation. Sometimes, specialized officials and craftsmen are called together to conduct primary research and textual criticism on it;

- Thirdly, it seamlessly combines functionality and appreciation. Convenience of storage and carry is always the first essence of design. There should be enough space between implements without influencing placement, taking and start and stop and opening and closing of case and drawer. Each grid's groove is dug according to implement's outline to make it stable in its position and to avoid collision, overturn and damage in displacement process. These are the most basic requirements for qualified design of Duobaoge;

- Fourthly, function of specific implement and nature of antique are weakened. After creative combination, we obtain not only visual effect unpossessed by a single implement, but also new relation between implements, thus endowing it with new significance;

- Fifthly, Duobaoge is space division art in essence. High and low structure at random after opening and mannered appearance after closing reflect the traditional idea of hiding ingenuity in plainness in essence, simply the "plainness" here is relative speaking;

- Sixthly, Duobaoge also reflects a kind of game play and interest. It contains mystery in layer upon layer of lattice drawers. Just like children playing hide-and-seek, people can obtain surprise from searching in constant opening and closing. Besides, although some objects are obviously before your eyes, you can not open the drawer or touch the object without finding the intrigue. It has the fun of puzzle solving, just like a toy. Moreover, the creation pleasure of taking part in design process on their own is irreplaceable by other activities to emperors.

\section{REFERENCES}

[1] Zhu Jiajin. Historical Data Series of Royal Workshop in Hall of Mental Cultivation(The first series)[M] Beijing: The Forbidden City Press, 2003/07.

[2] Zhang Rong. Historical Data Series of Royal Workshop in Hall of Mental Cultivation(The second series)[M] Beijing: The Imperial Palace Press, 2012/12.

[3] Zhang Rong. Historical Data Series of Royal Workshop in Hall of Mental Cultivation(The third series)[M] Beijing: The Imperial Palace Press, 2012/12.

[4] Zhang Rong. Historical Data Series of Royal Workshop in Hall of Mental Cultivation(The fourth series)[M] Beijing: The Imperial Palace Press, 2015/05.

[5] Tu Long(Ming). Kao Pan Yu Shi[M] Beijing: Gold Wall Press, 2011/07.

[6] Wen Zhenheng(Ming). Superfluous Things[M] Beijing: Zhonghua Book Company, 2012/05.

[7] Zhu Jiajin. Reduced Meals Record of Imperial Palace(Volume One and Volume Two)[M]Beijing: The Imperial Palace Press, 2014/05.

[8] The Palace Museum. Qing Dynasty Palace Packaging Art[M] Beijing: The Forbidden City Press, 2000/09.

[9] Lin Yongkuang. Qing Dynasty Palace Culture History[M] Shanghai: Shanghai Literature and Art Press, 2014/03.

[10] Zong Yishan. Research on Art Design of Stationary Accessories in Qianlong Period[D] Suzhou University, 2009.

[11] Chen Huixia. Qing Emperor Gaozong's Artistic Taste on Scholars Objects[J] The Imperial Palace Cultural Relic Monthly, 2013/9.

[12] Ji Ruoxin. Qianlong Dynasty Bamboo Filament Branch Tied Lotus Duobaoge Round Box in Display Files of Imperial Household of Qing Dynasty[J] The Imperial Palace Cultural Relic Monthly, 2013/12.

[13] Chinese University of Hong Kong, The First Public Records Office. Compiled Files of Royal Workshop in Imperial Household Department of Qing Dynasty Palace[M] Beijing: People's Publishing House, 2007. 